\title{
«Humor hat keine Grenzen»
}

\author{
Er zeichnet. Dabei schreibt er eigentlich. Nein, er erzählt Geschichten. Und oft sind \\ seine Geschichten Witze. Christophe Badoux ist Illustrator und Comic-Zeichner. \\ Und prominentes Mitglied des kleinen Künstler-Pools, der seit kurzem für die letzte \\ Seite der SÄZ verantwortlich zeichnet*. Skizzen eines Besuchs im Atelier von Badoux \\ im wilden Westen von Zürich.
}

Daniel Lüthi

Text und Fotos

\section{«lch werde versuchen, den Laienblick in}

\section{diese Fachzeitschrift zu bringen.»}

Mit Tischnachbar Marcel ist Badoux an einem Buchprojekt, das aus einem Blog entstanden ist. Christophe Badoux heisst nur «Badoux» hier, der Held des Blogs und Buches heisst «Stan the Hooli-

Das war einmal ein Pneulager. Jetzt ist es eine Ideenfabrik. Atelier-Gemeinschaft und kreative Brutstätte. Rund 15 Individualistinnen und Individualisten ar作 grossen Computer-Bildschirmen und hirnen. Entwerfen, zeichnen, scannen, schneiden, färben aus, animieren. Lassen Fantasien ihren Lauf, führen Spleens spazieren, bringen Leute zum Lachen und Genialitäten zum Gedeihen. Zum Mittagessen sitzen alle um den grossen Tisch in der Mitte. gan». Sie alle sind militante FCZ-Fans. «Wir stehen auf echter Fussball-Fan-Kultur mit Bier und Pyros, halten also wenig von Fussball als bravem FamilienEvent», sagt Badoux. Ein klares Bekenntnis. «Das hier ist das präsidiale Paar, Cillo und Heliane. Das Rot ihrer Haare ist die einzige Farbe, die wir nebst Blau und Weiss akzeptieren.» Marcel schmunzelt, das Gesicht von Badoux bleibt ernst, innerlich schmunzelt auch er. Die Stimmung ist gelöst. Aus dem Lautsprecher ertönt ziemlich laut «Jon Spencer Blues Explosion». Nicht jeder könnte dazu seriös arbeiten.

Ein Einbild-Witz für das Velojournal entsteht. «Manchmal ist die Idee sofort da, manchmal ist die Ideenfindung ein harter Knorz, ein unentwirrbarer Knäuel.» Badoux spricht in Bildern. Mit blauem Farbstift, der später unsichtbar wird, zeichnet er vor. Mit Pinsel und Tusche, und mit Filzstift und Lineal setzt er die definitiven Linien. Dann wird das Bild gescannt. Am Computer wird es anschliessend eingefärbt. Einen Text braucht es hier nicht. Das Bild erzählt den Witz selber.

Der Game-Designer nebenan taucht jeweils erst am Nachmittag auf, Martin, der Romand, ist auch noch nicht da, das Pult der Illustratoren-Legende Andrea ist als einziges aufgeräumt, Barbara animiert einen Trickfilm. «Abwechslung und Ablenkung tun
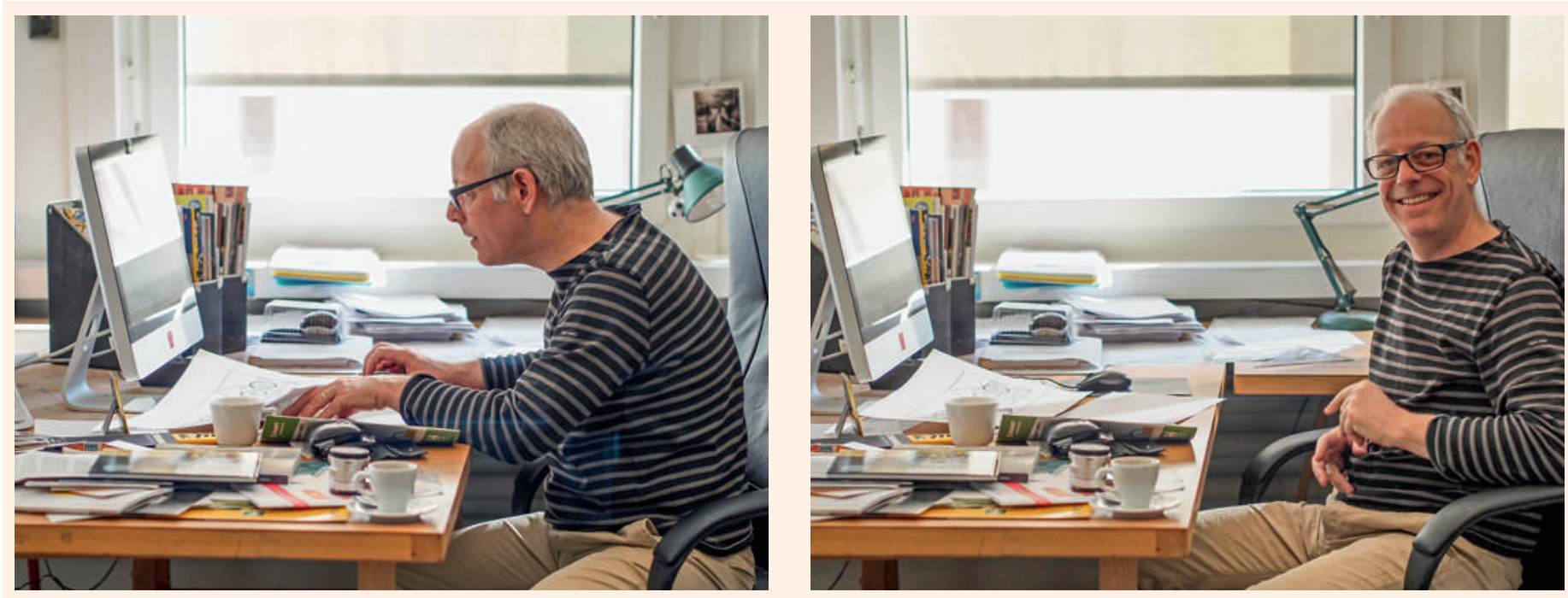


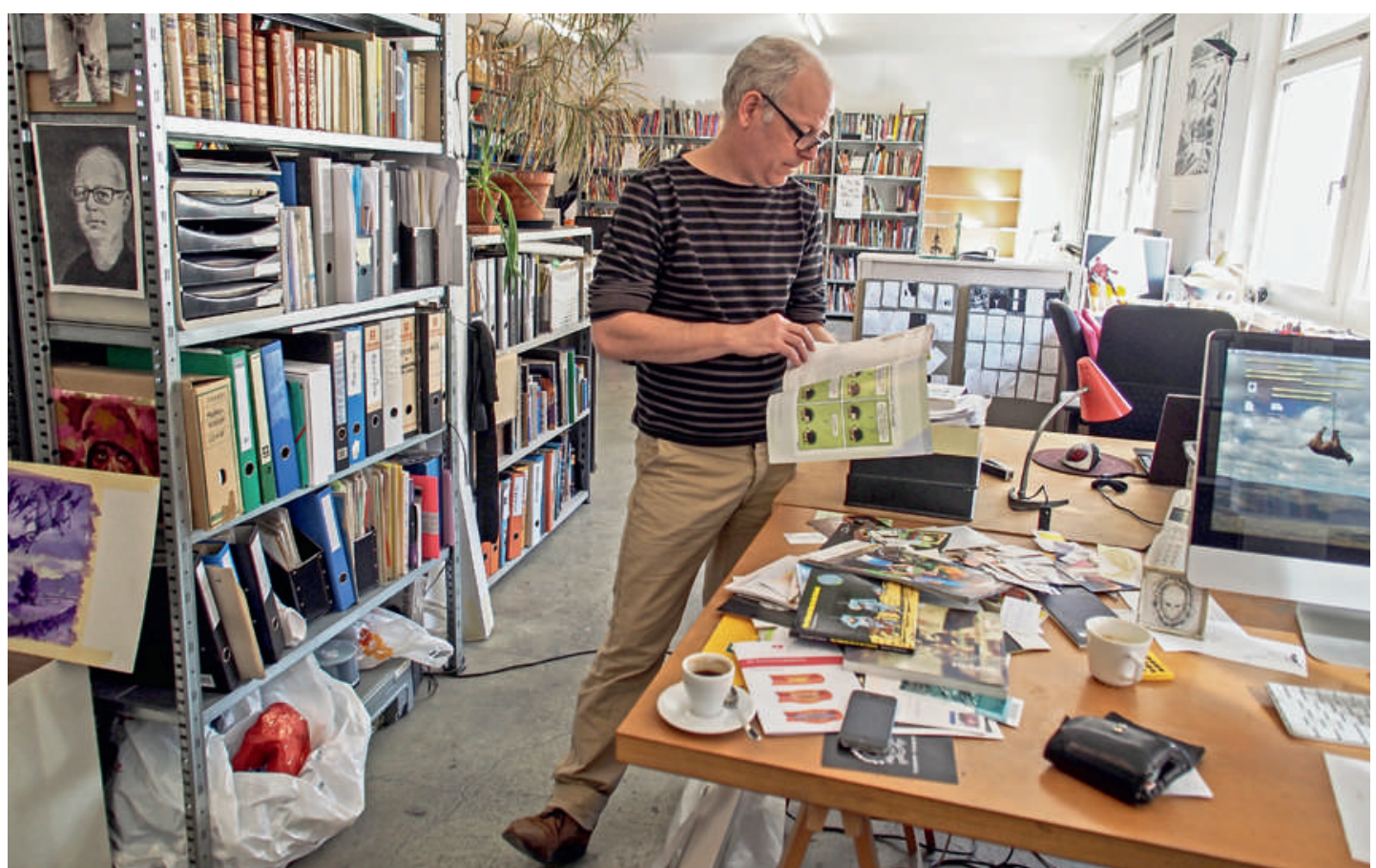

Badoux und die letzte Seite der SÄZ - ein neues Paar.

mir gut», sagt sie. «Zeichnen ist ein solitärer Job», sagt Badoux. Aber auch er geniesst die Präsenz der andern. Kati legt jeweils Musik auf, noch bevor sie den Mantel ablegt. «Soziopathen setzen dann

\section{«Heilige Kühe schlachte ich \\ besonders gern.»}

manchmal einen Kopfhörer auf», kommentiert Badoux. Am grossen Tisch in der Mitte macht eine Adhoc-Crew gerade die neue Nummer des Comic-Magazins «Strapazin» für den Versand an die Abonnen-

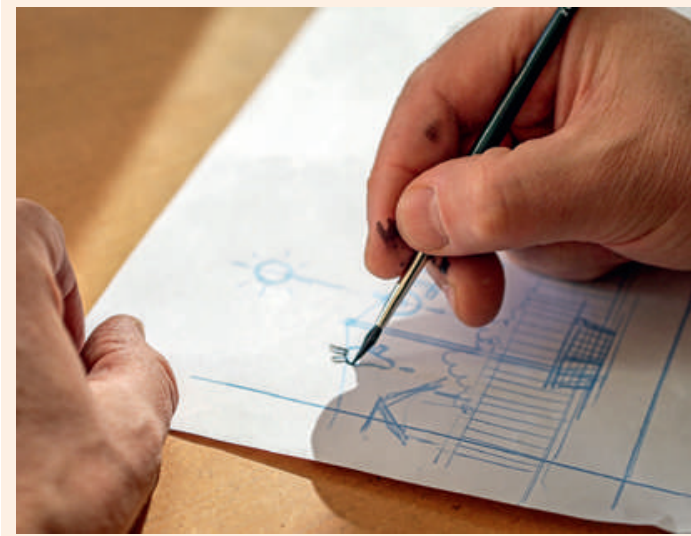

Die vorgezeichneten blauen Linien werden unsichtbar, es bleibt die Zeichnung mit den Pinselstrichen ... ten bereit. In einer älteren Nummer erzählen ein paar Dutzend Zeichnerinnen und Zeichner von ihrer Lieblings-Fernsehserie. Badoux erinnert sich in drei Bildern an 1972 und das Raumschiff Enterprise. Er schreibt von «Galaxien, die ich nie zuvor gesehen habe». $\mathrm{Zu}$ sehen ist im Vordergrund dreimal eine Dame mit bemerkenswerter Oberweite. Sie hat den damals achtjährigen Christophe offensichtlich besonders beeindruckt.

Bleiben wir bei den Astronauten. An ihrem Beispiel illustriert Badoux, warum er auch schon «Hergé der Schweiz» genannt worden ist: Das Titelbild seines Buchs «Mit dem Fahrrad durch die Galaxis» hat tatsächlich eklatante Parallelen zu Hergés Titelbild von Tim und Struppis Abenteuer «Schritte auf dem Mond». «Ligne claire» heisst die auffälligste Parallele

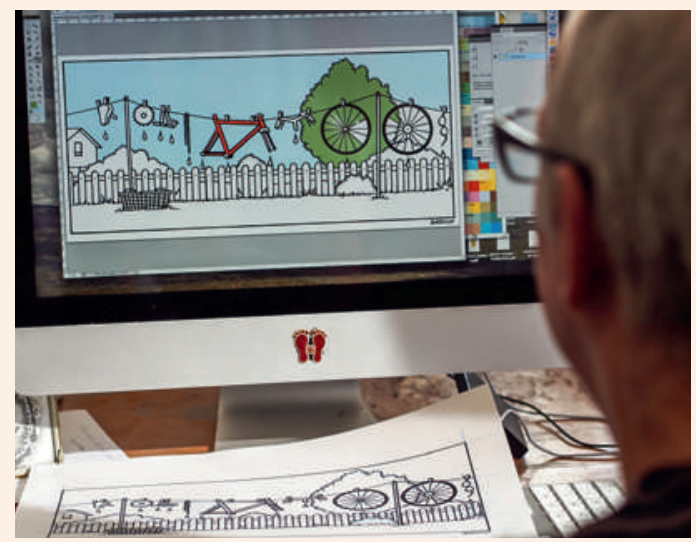

... sie wird eingescannt und per Computer eingefärbt. 


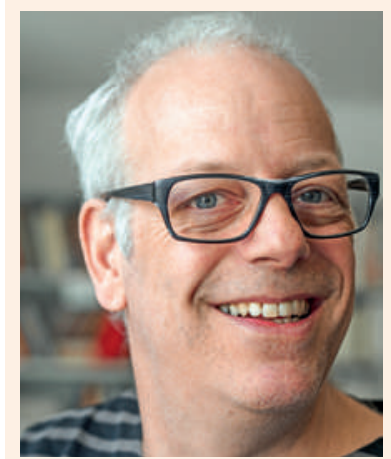

Christophe Badoux wurde 1964 in Barbourville (Kentucky/USA) geboren, wo sein Vater an der Universität unterrichtete. Als knapp Zweijähriger kam Badoux zurück in die Schweiz. Zuerst nach Zuoz, dann nach Greifensee, wo er aufwuchs. In Zürich besuchte er die Kunstgewerbeschule, wo er nach dem Vorkurs die Grafik-Fachklasse absolvierte. 1986 bis 1994 lebte er in Paris, wo er während einiger Jahre als Werbegrafiker arbeitete. Badoux zeichnet für verschiedene Zeitungen und Zeitschriften, und er veröffentlichte mehrere Comic-Bände, so 2006 «Fatmas fantastische Reise», wo er die seltene Gehirnkrankheit Moyamoya thematisiert, oder 2008 eine Biographie über Paul Klee. Seit 2003 arbeitet Badoux als Dozent an der Abteilung Design und Kunst der Hochschule Luzern. Badoux ist verheiratet mit der Musikerin Nadja Zela und Vater von zwei Kindern. Er lebt mit seiner Familie in Zürich. in der Fachsprache. «Mit Hergé verglichen zu werden, ist eine grosse Ehre für mich», sagt Badoux, «ich zeichne ähnlich wie er, ja, aber neben seiner filigranen Technik wirken meine Zeichnungen wie Holzschnitte, da liegen Welten dazwischen. Trotzdem: Ich zitiere ihn gerne. Und Imitation gilt in Japan ja geradezu als Ehrerbietung.»

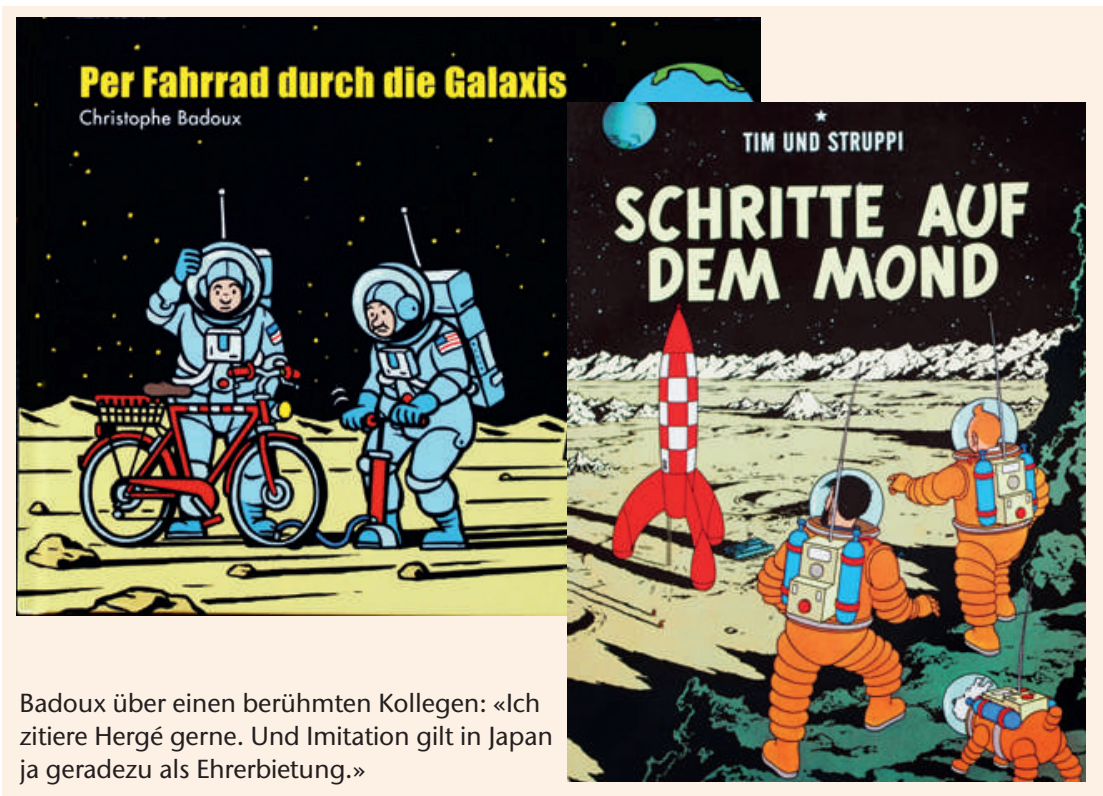

Der Mann hat einen weiten Blick. In der Regel versucht er, die Dinge von oben zu betrachten. Oder von der Kehrseite her. Und meistens sucht er ein konkretes Bild, um ein abstraktes Phänomen zu erklären, einen grossen Begriff zu illustrieren oder einen Vorgang zu kommentieren. Ein Beispiel: Wie soll einer das Thema Antibiotika darstellen? «Ich habe mir erzählen lassen, was Antibiotika tun», erzählt Badoux. «Was ich hörte, ist eine Kriegsberichterstattung: Da kämpfen die Guten gegen die Bösen, die Antibiotika gegen die Bakterien.» Das Bild, das daraus entstand, zeigt in klassischer Manier ein Schlachtfeld mit Kanonen und wehenden Fahnen.

Apropos Gesundheitsthemen: Was reizt Badoux daran? «Ich hole Hochtrabendes gerne auf den Boden herunter. So werde ich versuchen, den Laienblick in diese Fachzeitschrift zu bringen und Spezialistenthemen verständlich zu machen. Psychiater zum Beispiel haben ein unglaubliches Witz-Potential.» Schnell wird der Psychiater zum Seelen-Klempner und damit zum Handwerker, der etwas Konkretes tut. Und die Geschichte wird zum Comic-Strip. Dieser hier wurde bereits veröffentlicht. «Ins Französische liess sich das Wortspiel übrigens nicht übersetzen und ich musste einen anderen Witz draus machen», fügt Badoux bei. Und mit Blick in die $\mathrm{Zu}$ kunft: «Ich freue ich mich auch darauf, mich über die Pharmaindustrie lustig zu machen. Es ist doch legitim, das wirtschaftliche Denken, das alles rechtfertigt, zu hinterfragen, oder? Übrigens: Heilige Kühe schlachte ich besonders gern.»

Gibt es hier für einen Comiczeichner Tabus? Badoux überlegt. «Ich wüsste nicht was», sagt er. «Über Patientenverfügungen habe ich auch schon einen Witz gemacht. Humor hat keine Grenzen. Und gerade der Gesundheitsbereich ist ja eine tabufreie Zone. Natürlich ist alles eine Frage des Geschmacks. Hundert Prozent der Leute erreiche ich nie. Das schafft nur Roger Federer. Es gibt Leute, die sehr wenig vertragen. Und es gibt auch dumme Leute. Aber selbstverständlich habe ich meine eigenen moralischen Barrieren. Einen rassistischen Witz zum Beispiel würde ich nicht machen. Oder nur auf der zweiten oder dritten Ebene. Aber das ist schwierig zu erklären.»

$\mathrm{Zu}$ zeichnen wäre es für Badoux wahrscheinlich einfacher.

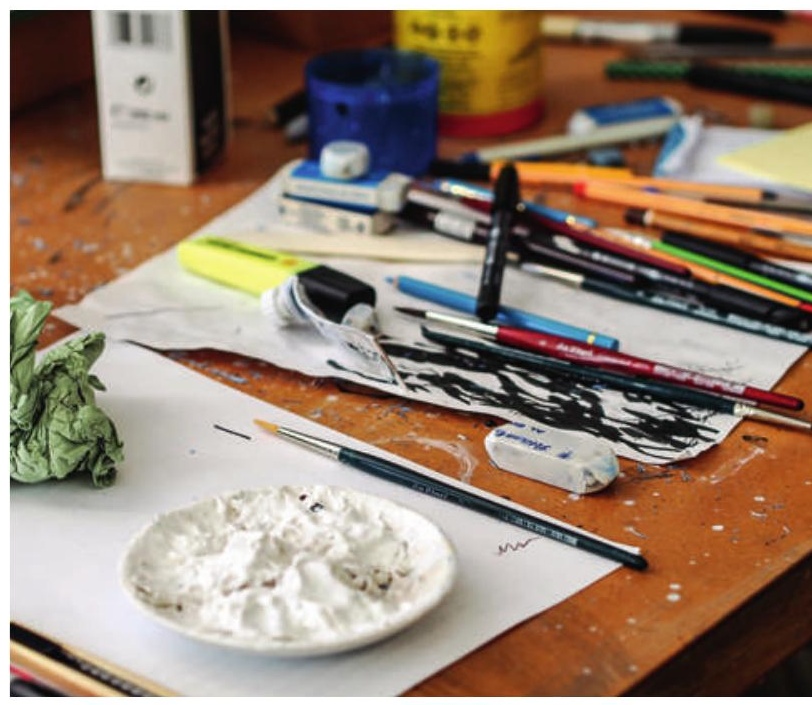

